\title{
Sur un théorème de $S$. Saks concernant les suites infinies de fonctions continues
}

\author{
par \\ W. Sierpiński（Warszawa)
}

Dans mon livre Hypothèse du continu, Warszawa-Lwów 1934 (Monografie Matematyczne IV) à la p. 47. on trouve le théorème 8 suivant:

THÉORÈme. Si un ensemble $Q$ jouit de la propriété $\mathrm{L}$, il existe une suite infinie de fonctions d'une variable réelle $f_{1}(x), f_{2}(x), \ldots$ continues, uniformément bornées et telles que pour chaque suite infinie oroissante d'indioes $m_{1}, m_{2}, \ldots$, la suite $f_{m_{1}}(x), f_{m_{2}}(x), \ldots$ est convergente tout au plus en une infinité dénombrable de points de Q.

(On dit qu'un ensemble $E$ jouit de la propriété L (de Lusin) si tout ensemble parfait non dense (donc aussi tout ensemble de 1-re catégorie) contient un ensemble au plus dénombrable de points de $E$.) Au renvoi ${ }^{1}$ ) on lit que ce théorème et sa démonstration sont dus à $\mathrm{S}$. Saks.

Le commencement de la démonstration est le suivant. On voit sans peine que l'on peut définir (par induction) une suite infinie de fonctions continues d'une variable réelle $f_{x}(x), f_{2}(x), \ldots$ telle que l'on ait $0 \leqslant f_{n}(x) \leqslant 1$ pour tout $n$ naturel et pour $x$ réel, et qui remplisse en outre la condition suiviante:

(1) quel que soit $n=1,2, \ldots$, il existe pour tout intervalle $I$ de longueur $>1 / n\left({ }^{(}\right)$et pour tout indice $k<n$ un nombre réel $x \in I$ tel que

$$
\left|f_{n}(x)-f_{k}(x)\right| \geqslant \frac{1}{2} .
$$

Or, nous allons montrer que chaque suite $f_{n}(x)(n=1,2, \ldots)$ de ce genre (qui est uniformément bornée par définition) satisfait à la thèse du théorème.

En ce qui concerne la démonstration ultérieure, M. Sunyer Balaguer a remarqué qu'elle n'est pas correcte, car elle utilise une proposition fausse, d'après laquelle il serait possible d'extraire de toute suite infinie de fonctions continues et uniformément bornées convergente dans un ensemble dénombrable $D$ une suite qui converge uniformément dans $D$.

(1) Dans mon livre on lit $<1 / n$, ce qui est sans doute une faute d'impression. 
(Il est évident que ceci est impossible pour la suite $g_{n}(x)=x^{n}(n=1,2, \ldots)$ qui converge dans l'ensemble $D=\left\{\frac{1}{2}, \frac{2}{3}, \ldots, k /(k+1), \ldots\right\}$ et où les fonctions $g_{n}(x)$ sont continues et uniformément bornées dans $D$ ).

Stazislas Saks été tué par la Gestapo en novembre de 1942 et ses manuscrits n'existent plus. J'ai perdu moi-même dans les flarnmes ma bibliothèque et mes archives en 1944. Il est donc impossible d'établir aujourd'hui quelle était la démonstration de $\mathrm{S}$. Saks. En tout cas il est étonnant que grâce à M. Sunyer Balaguer, l'on n'ait trouvé la faute, que 23 années après l'apparition de la première édition de mon livre.

Heureusement, non seulement le théorème de S. Saks est rrai, mais même la suite $f_{n}(x) \quad(n=1,2, \ldots)$ définie plus haut satisfait à la thèse de son théorème. Le but de cette Note est de le prouver. Tout d'abord, j'expliquerai comment on peut définir par induction la suite $f_{n}(x)$ $(n=1,2, \ldots)$ satisfaisant à la condition (1).

Soit $f_{1}(x)=0$ pour $x$ réels. Soit maintenant $n$ un indice $>1$ et supposons que nous ayons déjà défini les fonctions $f_{k}(x)$ pour $k<n$. Soit $j$ un entier et $k$ un nombre naturel $<n$. Posons

$$
f_{n}\left(\frac{j}{2 n}+\frac{k}{2 n^{2}}\right)=f_{k}\left(\frac{j}{2 n}+\frac{k}{2 n^{2}}\right) \pm \frac{1}{2}
$$

en prenant le signe + si

et le signe - si

$$
f_{k}\left(\frac{j}{2 n}+\frac{k}{2 n^{2}}\right) \leqslant \frac{1}{2}
$$

$$
f_{k}\left(\frac{j}{2 n}+\frac{k}{2 n^{2}}\right)>\frac{1}{2} \text {. }
$$

La fonction $f_{n}(x)$ est ainsi définie aux points $j / 2 n+k / 2 n^{2}$, où $j$ est un entier et $k$ un nombre naturel $<n$, et on peut la définir pour tous les $x$ réels de sorte qu'elle soit linéaje entre deux points consécutifs pour lesquels nous l'avons définie. On vérifie sans peine que la suite $f_{n}(x)$ $(n=1,2, \ldots)$ ainsi obtenue satisfait à la condition (1).

Soit maintenant $m_{1}, m_{2}, \ldots$ une suite infinie croissante de nombres naturels. Je démontrerai que l'ensemble $C$ des points de convergence de la suite infinie $f_{m_{i}}(x)(i=1,2, \ldots)$ est de première catégorie. Supposons, en effet, que l'ensemble $C$ ne soit pas de première catégorie. Les fonctions $f_{m_{i}}(x)(i=1,2, \ldots)$ étant continues, l'ensemble $O$ est un $F_{\sigma \delta}$ (voir [1], p. 397), donc il est un ensemble borelien. Il en résulte que l'ensemble $C$ jouit de la propriété de Baire (voir par exemple [2], p. 56), donc il est de la forme $C=G-P+R$, où $G$ est ouvert et $P$ et $R$ sont des ensembles de 1-re catégorie ([2], p. 54). L'ensemble $O$ n'étant pas, par hypothèse, de 1-re catégorie, il en résulte que l'ensemble $G$ n'est pas vide, étant ouvert, il contient un intervalle $d_{0}$. On a done $d_{n}-P C C$. L'ensemble $P$ étant de 1-re catégorie, on a $P=N_{1}+N_{2}+\ldots$, ò̀ $N_{i}(i=1,2, \ldots)$ sont des ensembles non denses.

La fonction $f_{m_{1}}(x)$ étant continue, il existe un intervalle $\delta_{1} \subset d_{0}$ tel que l'oscillation de la fonction $f_{m_{1}}(x)$ est $<\frac{1}{6}$ dans $\delta_{1}$. Posons $p_{1}=1$. L'ensemble $N_{1}$ étant non dense, il existe un intervalle $d_{1} C \delta_{1}$ tel que $N_{1} d_{1}=0$

Je définirai maintenant par induction deux suites infinies d'intervalles fermés $\delta_{1}, \delta_{2}, \ldots$ et $d_{1}, d_{2}, \ldots$ et une suite infinie d'indices croissants $p_{1}, p_{2}, \ldots$

Soit $k$ un nombre naturel et supposons que nous ayons déjà défini les intervalles $\delta_{k}$ et $d_{k}$ et l'indice $p_{k}$. La suite infinie $m_{1}, m_{2}, \ldots$ étant croissante, il existe un indice $s>p_{k}$ tel que le nombre $1 / m_{s}$ est plus petit que la longueur de l'intervalle $d_{k}$. Posons $p_{k+1}=\delta$. La longueur de l'intervalle $d_{k}$ est done $>1 / m_{p_{k+1}}$ et, d'après (1) il existe dans $d_{k}$ un nombre réel $y$ tel que

$$
\left|f_{m_{p_{k+1}}}(y)-f_{m_{p_{k}}}(y)\right| \geqslant \frac{1}{2}
$$

La fonction $f_{m_{p_{k+1}}}(x)$ étant continue au point $y$, et vu que $y \in d_{k}$, il existe un intervalle $\delta_{k+1} \subset d_{k}$ contenant $y$ et tel que l'ascillation de la fonction $f_{m_{p_{k+1}}}(x)$ est $<\frac{1}{8}$ dans $\delta_{k+1}$. Or, l'ensemble $N_{k+1}$ étant non dense, il existe un intervalle $d_{k+1} \subset \delta_{k+1}$ tel que $d_{k+1} N_{k+1}=0$.

Les intervalles $d_{0} \supset \delta_{1} \supset d_{1} \supset \delta_{2} \supset d_{2} \supset \ldots$ et les indices $p_{1}<p_{2}<\ldots$ sont ainsi définis par induction. $\Pi 1$ existe donc un nombre $t_{0} \in d_{0} \delta_{1} d_{1} \delta_{2} d_{2} \ldots$ D'après $d_{i} N_{i}=0$ pour $i=1,2, \ldots$ on a donc (d'après $P=N_{1}+N_{2}+\ldots$ ), $t \in d-P C C$. La suite $f_{m_{p_{k}}}(t)(k=1,2, \ldots)$ est donc convergente. Or, soit $k$ un nombre naturel domné. L'oscillation de la fonction $f_{m_{p_{k}}}$ est $<\frac{1}{8}$ dans $\delta_{k}$ et, à plus forte raison, dans $\delta_{k+1}$, et l'oseillation de la fonction $f_{m_{p_{k+1}}}$ est $<\frac{1}{8}$ dans $\delta_{k+1}$. Or, comme $y \in \delta_{k+1}$ et

$$
\begin{aligned}
\frac{1}{2} & \leqslant\left|f_{m_{p_{k+1}}}(y)-f_{m_{p_{k}}}(y)\right| \\
& \leqslant\left|f_{m_{p_{k+1}}}(y)-f_{m_{p_{k+1}}}(x)\right|+\left|f_{m_{p_{k+1}}}(x)-f_{m_{p_{k}}}(x)\right|+\left|f_{m_{p_{k}}}(x)-f_{m_{p_{k}}}(y)\right|
\end{aligned}
$$

pour $k=1,2, \ldots, x \in \delta_{k+1}$, on trouve que

$$
\left|f_{m_{p_{k+1}}}(x)-f_{m_{p_{k}}}(x)\right|>\frac{1}{4} \quad \text { pour } \quad x \in \delta_{k+1} .
$$

Comme $t \in \delta_{k+1}$, pour $k=1,2, \ldots$, on a done

$$
\left|f_{m_{p_{k+1}}}(t)-f_{m_{p_{k}}}(t)\right|>\frac{1}{4} \quad \text { pour } \quad k=1,2, \ldots
$$

et la suite $f_{m_{i}}(t)(i=1,2, \ldots)$ est divergente. 
L'hypothèse que l'ensemble $O$ n'est pas de 1-re catégorie implique donc une contradiction.

Donc, l'ensemble $O$ est de 1-re catégorie et, vu la propriété de l'ensemble $Q$, il en résulte que l'ensemble $Q O$ est au plus dénombrable. Le théorème de $\mathbf{S}$. Saks se trouve ainsi démontré.

En publiant cette démonstration je rends hommage à la mémoire de l'éminent mathématicien, Stanislas Saks, qui a péri il y a 15 ans.

Le théorème de $\mathrm{S}$. Saks résulte aussi tout de suite du théorème suivant qui m'a étë communiqué par R. Sikorski après que j'eusse rédigé la démonstration qui précède.

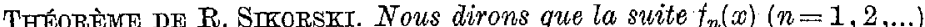
de fonctions continues (d'une variable réelle) jouit de la propriété $\mathrm{S}$, s'it existe un nombre $a>0$ et une suite infinie de nombres positifs ' $a_{1}, a_{2}, \ldots$ convergente vers 0 et telle qu'il existe pour tout intervalle de longueur $>a_{n}$ et tout nombre naturel $k<n$ un nombre $x$ de cet intervalle, tel que

$$
\left|f_{n}(x)-f_{k}(x)\right|>a \text {. }
$$

THÈse. L'ensemble des points de convergence d'une suite $f_{n}(x)$ (n $=1,2, \ldots)$ jouissant de la propriété $\mathrm{S}$ est de 1-re catégorie (sur la droite).

Démonstration (de R. Sikorski). L'ensemble

$$
A_{k, m, n}=E_{x}\left[\left|f_{m+n}(x)-f_{m}(x)\right|>1 / k\right]
$$

est ouvert. Soit $t /$ un nombre naturel tel que $a>1 / k$. Il résulte de la propriété S que l'ensemble ouvert

$$
\sum_{n=1}^{\infty} A_{k, m, n}
$$

est dense sur la droite. L'ensemble

$$
\prod_{m=1}^{\infty} \sum_{n=1}^{\infty} A_{k, m, n}
$$

est donc résiduel (c'est-à-dire complémentaire d'un ensemble de 1-re cartégorie). Done l'ensemble

$$
A=\sum_{k=1}^{\infty} \prod_{m=1}^{\infty} \sum_{n=1}^{\infty} A_{k, m, n}
$$

l'est aussi, en tant que sur-ensemble d'un ensemble résiduel. Or, comme

$$
A=E_{x}\left[\sum_{k} \prod_{m} \sum_{n}\left(\left|f_{m+n}(x)-f_{m}(x)\right|>1 / k\right)\right],
$$

A est l'ensemble des points de divergence de la suite $f_{n}(x)(n=1,2, \ldots)$, et le théorème de $R$. Sikorski se trouve démontré.
$\Pi$ est érident que tonte suite infinie extraite d'une suite jouissant de la propriété $\mathbf{S}$ jouit de cette propriété.

Il existe des suites infinies uniformément bornées de fonctions contimues $f_{n}(x)(n=1,2, \ldots)$ jouissant de la propriété $s:$ telle est par exemple, ce qu'on vérifie sans peine (pour $a=1-1 / 2 / 2 a_{n}=2 \pi / 2^{n}$ ), la suite $f_{n}(x)=\sin 2^{n} x$. Telle est aussi toute suite satisfaisant à la condition (1).

Le théorème de $R$. Sikorski entraine donc le théorème de $\mathrm{S}$. Saks. Varsovie, le 24 octobre 1957.

\section{Travanx cités}

[1] F. Hausdorff, Grundxüge der Mengenlehre, Leipzig 1914.

[2] O. Kuratowski, Topologie $I, 3$-ème édition, Warszawa 1952.

Reģu par la Rédaction le 28.1.1958 